Article

\title{
Synphilin-1 Interacts with AMPK and Increases AMPK Phosphorylation
}

\author{
Tianxia Li ${ }^{1,2}$, Jingnan Liu ${ }^{2,+} \oplus$, Gongbo Guo ${ }^{1}$, Bo Ning ${ }^{1,3}$, Xueping Li ${ }^{1,3}$, Guangjing Zhu ${ }^{1}$, \\ Dejun Yang ${ }^{1}{ }^{(}$, Timothy H. Moran ${ }^{1}$ and Wanli W. Smith ${ }^{1, *(\mathbb{D})}$ \\ 1 Department of Psychiatry, Johns Hopkins University School of Medicine, Baltimore, MD 21205, USA; \\ Tianxia_li@dfci.harvard.edu (T.L.); gguo1@jhmi.edu (G.G.); bning2@jhmi.edu (B.N.); \\ xuepingli457@gmail.com (X.L.); guangjingzhu@hotmail.com (G.Z.); yangdjfeeding@gmail.com (D.Y.); \\ tmoran@jhmi.edu (T.H.M.) \\ 2 Department of Pharmaceutical Sciences, University of Maryland School of Pharmacy, \\ Baltimore, MD 21201, USA; liujn@shanghaitech.edu.cn \\ 3 Institute of Obesity and Metabolic Diseases, School of Clinical Medicine, Xi'an Medical Collage, \\ Xi'an 71000, China \\ * Correspondence: wsmith60@jhmi.edu; Tel.: +1-410-6146268 \\ + Present address: School of Life Science and Technology, ShanghaiTech University, Shanghai 201210, China.
}

Received: 6 May 2020; Accepted: 9 June 2020; Published: 18 June 2020

check for updates

\begin{abstract}
A role for the cytoplasmic protein synphilin-1 in regulating energy balance has been demonstrated recently. Expression of synphilin-1 increases ATP levels in cultured cells. However, the mechanism by which synphilin-1 alters cellular energy status is unknown. Here, we used cell models and biochemical approaches to investigate the cellular functions of synphilin-1 on the AMP-activated protein kinase (AMPK) signaling pathway, which may affect energy balance. Overexpression of synphilin-1 increased AMPK phosphorylation (activation). Moreover, synphilin-1 interacted with AMPK by co-immunoprecipitation and GST (glutathione S-transferase) pull-down assays. Knockdown of synphilin-1 reduced AMPK phosphorylation. Overexpression of synphilin-1 also altered AMPK downstream signaling, i.e., a decrease in acetyl CoA carboxylase (ACC) phosphorylation, and an increase in p70S6K phosphorylation. Treatment of compound $C$ (an AMPK inhibitor) reduced synphilin-1 binding with AMPK. In addition, compound C diminished synphilin-1-induced AMPK phosphorylation, and the increase in cellular ATP (adenosine triphosphate) levels. Our results demonstrated that synphilin-1 couples with AMPK, and they exert mutual effects on each other to regulate cellular energy status. These findings not only identify novel cellular actions of synphilin-1, but also provide new insights into the roles of synphilin-1 in regulating energy currency, ATP.
\end{abstract}

Keywords: synphilin-1; AMPK; ATP; energy balance; protein interaction

\section{Introduction}

Recent studies in transgenic animal models suggest that synphilin-1 is involved in regulating energy homeostasis [1,2]. Synphilin-1, a 919-amino acid protein, is expressed in various tissues and has high expression in the brain [3-5]. The full range of biological functions of synphilin- 1 is not clear. Previous studies have shown that synphilin-1 can interact with multiple proteins (e.g., alpha-synuclein) and is involved in the pathogenesis of Parkinson's disease (PD) in relation to protein aggregation $[3,4,6-14]$, suggesting a putative role of synphilin-1 in PD. We and others have demonstrated that synphilin-1 has neurotrophic and neuroprotective effects [15-19]. The recent studies of human synphilin-1 in transgenic flies and mice have demonstrated that synphilin-1 induces 
hyperphagia and obesity [1,2]. In cultured cells, expression of synphilin-1 increased cellular ATP levels [2]. However, it is not clear how synphilin-1 alters the energy balance.

AMP-activated protein kinase (AMPK, a serine/threonine kinase) is an integrator of regulatory signals that modulate the systemic and cellular energy status in response to environmental nutritional changes [20-25]. Disruption of the AMPK pathway leads to metabolic dysregulation, resulting in various metabolic disorders. AMPK regulates a wide range of metabolic pathways [26,27] and has become a therapeutic target for the treatment of type II diabetes mellitus and obesity [27]. In the hypothalamus, AMPK is a master regulator of energy homeostasis by integrating nutrient and hormonal signals [24,28]. A decrease in hypothalamic AMPK activity is associated with suppression of feeding behavior, while activation of AMPK leads to increased food intake. Insulin and leptin suppress hypothalamic AMPK activity [29]. Orexigenic peptides such as the gut-derived hormone ghrelin and the neuropeptide agouti-related peptide [29] stimulate hypothalamic AMPK activity to increase food intake $[30,31]$.

AMPK and synphilin-1 are both predominantly expressed in the cytosol. However, whether synphilin-1 and AMPK affect one another in controlling energy balance is not known. Thus, in this study, we used molecular biology tools and biochemical approaches to explore the relationship and functional consequences of the interactions between synphilin-1 and AMPK. Our findings identified a novel interaction between synphilin-1 and AMPK, which regulates cellular ATP levels. Therefore, we conclude that synphilin-1 interacts with AMPK and modulates energy homeostasis in cells.

\section{Results}

\subsection{Synphilin-1 Increased AMPK Phosphorylation}

Increased AMPK $\alpha$ phosphorylation at threonine 172 reflects increased AMPK activation [32]. To assess whether synphilin-1 alters AMPK phosphorylation, HEK 293 cells were transiently transfected with human synphilin-1, followed by a Western blot analysis using anti-phospho-AMPK $\alpha$ antibodies (at the threonine residue 172). Expression of human synphilin-1 significantly increased AMPK phosphorylation at threonine residue 172, up to $\sim 3$ fold (Figure 1A,B) compared with vector cells.

Moreover, synphilin-1 also altered AMPK downstream targets, i.e., it decreased acetyl CoA carboxylase (ACC) phosphorylation (Figure 1C,D), and increased p70 ribosomal S6 kinase (p70S6K) phosphorylation (Figure 1C,E). There were no significant differences detected in total AMPK $\alpha, A C C$ and p70S6K levels between cells expressing synphilin-1 and just the vector (Figure 1A,C). These results suggest that synphilin-1 regulates AMPK-linked signaling pathways. 

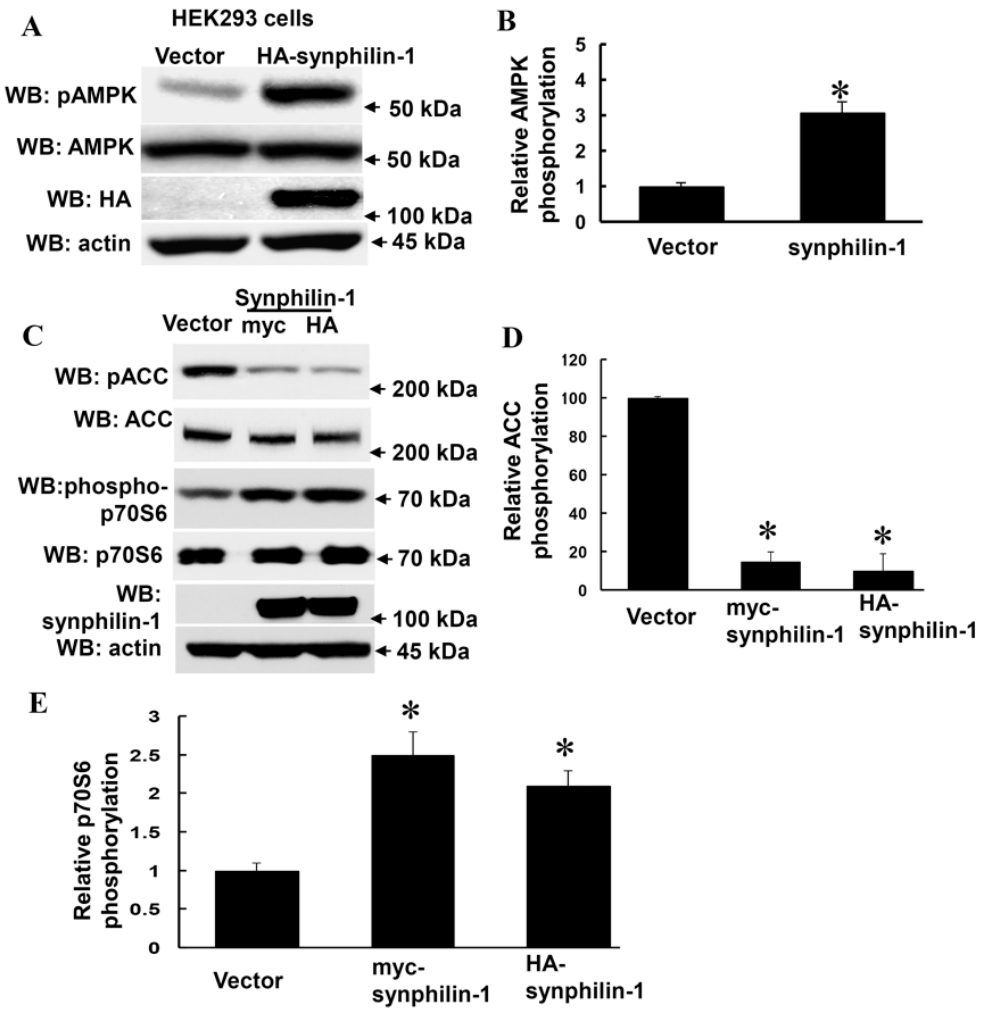

Figure 1. Overexpression of Synphilin-1 increased AMPK phosphorylation. (A) HEK293 cells were transfected with myc-synphilin-1 and vector for $48 \mathrm{~h}$. (A,B) Cell lysates from HEK293 cells expressing human synphilin-1 were subjected to immunoblot analysis using anti-HA, anti-AMPK $\alpha$, anti-phosphorylation AMPK $\alpha$ (pAMPK), and anti-actin antibodies. (A). Representative blots from three separated experiments. (B) Quantification of phosphorylated AMPK $\alpha$ levels normalized to total AMPK $\alpha$ levels. (C-E) Cell lysates from HEK293 cells expressing human synphilin-1 were subjected to immunoblot analysis using anti-ACC $\alpha$, anti-phospho-ACC $\alpha$ (pACC), anti-phospho-p70S6, anti-p70S6, anti-synphilin-1, and anti-actin antibodies. (C) Representative blots from three separated experiments. (D,E). Quantification of phosphorylated ACC $\alpha$ and p70S6 levels normalized to total ACC $\alpha$ and p70S6 levels. ${ }^{*} p<0.05$ by ANOVA followed by Tukey's post-hoc test, vs. vector control cells.

\subsection{Synphilin-1 Binds AMPK}

To further investigate the relationship between synphilin-1 and AMPK, we tested whether synphilin-1 interacts with AMPK. Synphilin-1 is a cytoplasmic protein and has been shown to interact with other cytoplasmic proteins $[7,13,19]$. We transfected myc-tagged synphilin-1 into HEK293 cells followed by co-immunoprecipitation assays. Myc-tagged synphilin-1 was immunoprecipitated using anti-myc antibodies, and endogenous AMPK could be detected by anti-AMPK immunoblotting (Figure 2A, top). Conversely, endogenous AMPK was immunoprecipitated using anti-AMPK antibodies, and myc-tagged synphilin- 1 could be detected by anti-myc immunoblotting (Figure 2A, middle). To validate this interaction, GST pull-down assays were performed. Pull-down of GST-synphilin-1 also could pull-down AMPK (Figure 2B). 


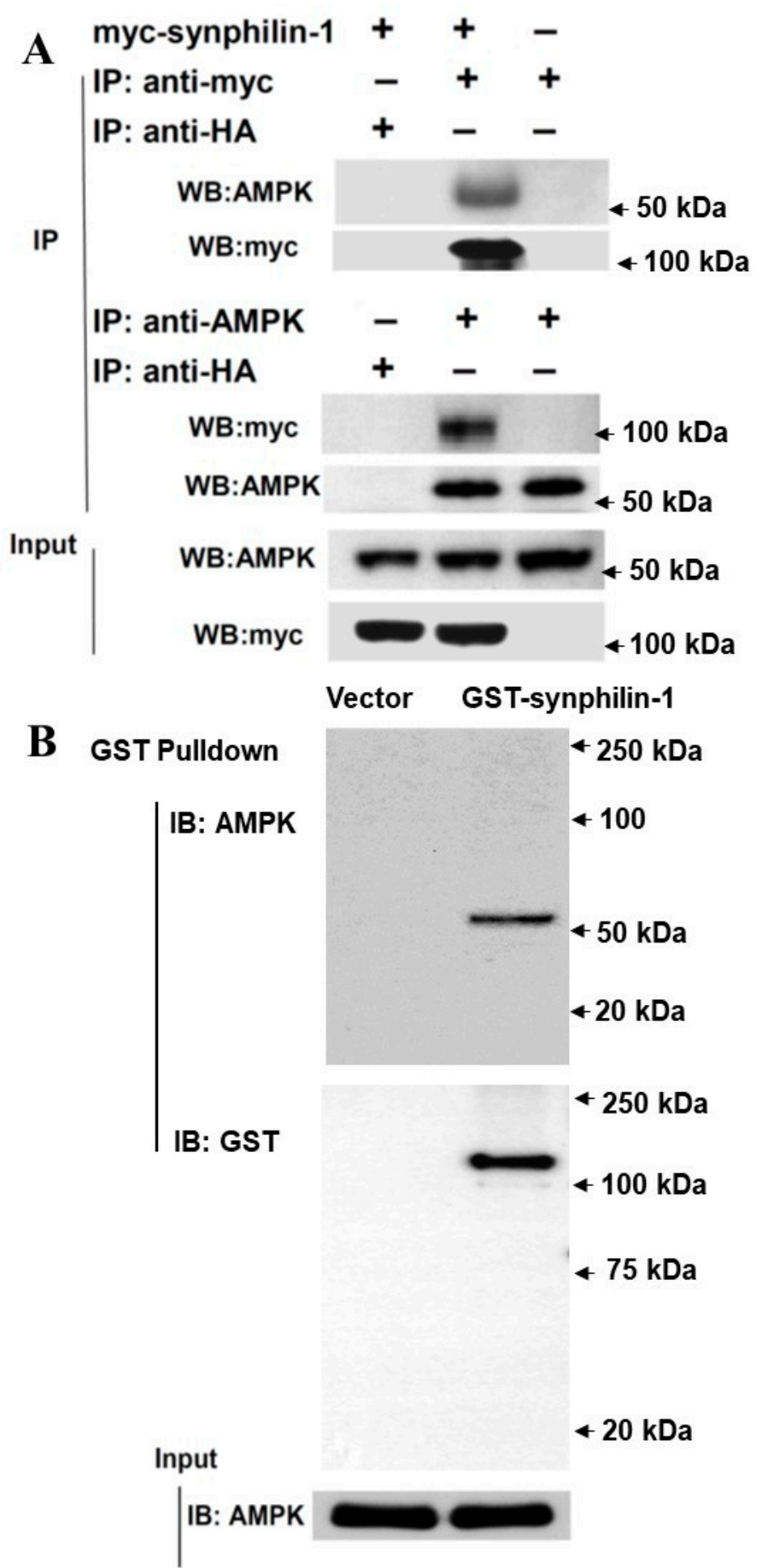

Figure 2. Synphilin-1 interacts with AMPK. (A). Lysates prepared from HEK 293 cells transfected with myc-tagged human synphilin-1 cDNA were subjected to IP with anti-myc, anti-HA, or anti-AMPK, followed by anti-AMPK, and anti-myc immunoblotting. Top: Anti-myc antibody precipitated myc-tagged synphilin-1 and AMPK. Middle: Anti-AMPK antibody precipitated AMPK and myc-tagged synphilin-1. Bottom: input blots showing the equal protein loading. (B). GST pull-down assays. Top: GST-beads were used to pull-down GST-tagged synphilin-1 and then followed by immunoblotting using anti-AMPK and anti-GST antibodies. Bottom: immunoblotting analysis of input lysates using anti-AMPK antibodies. 
To further map the interaction regions of synphilin-1 with AMPK, HA-tagged truncated synphilin-1 constructs were transfected into HEK 293 cells. The cell lysates were subjected to anti-HA co-IP, followed by Western blot analysis using anti-AMPK antibody. The F1B (1-246 aa), F1C (1-349 aa), F3 (550-659 aa), F4 (550-769 aa), and F6 (550-919 aa) sites interacted with AMPK (Figure 3). In contrast, F1A (1-108 aa), F2 (350-550 aa), and F7 (770-919 aa) did not interact with AMPK. These results indicated that two synphilin-1 regions interact with AMPK: 108-246 aa and 550-769 aa.

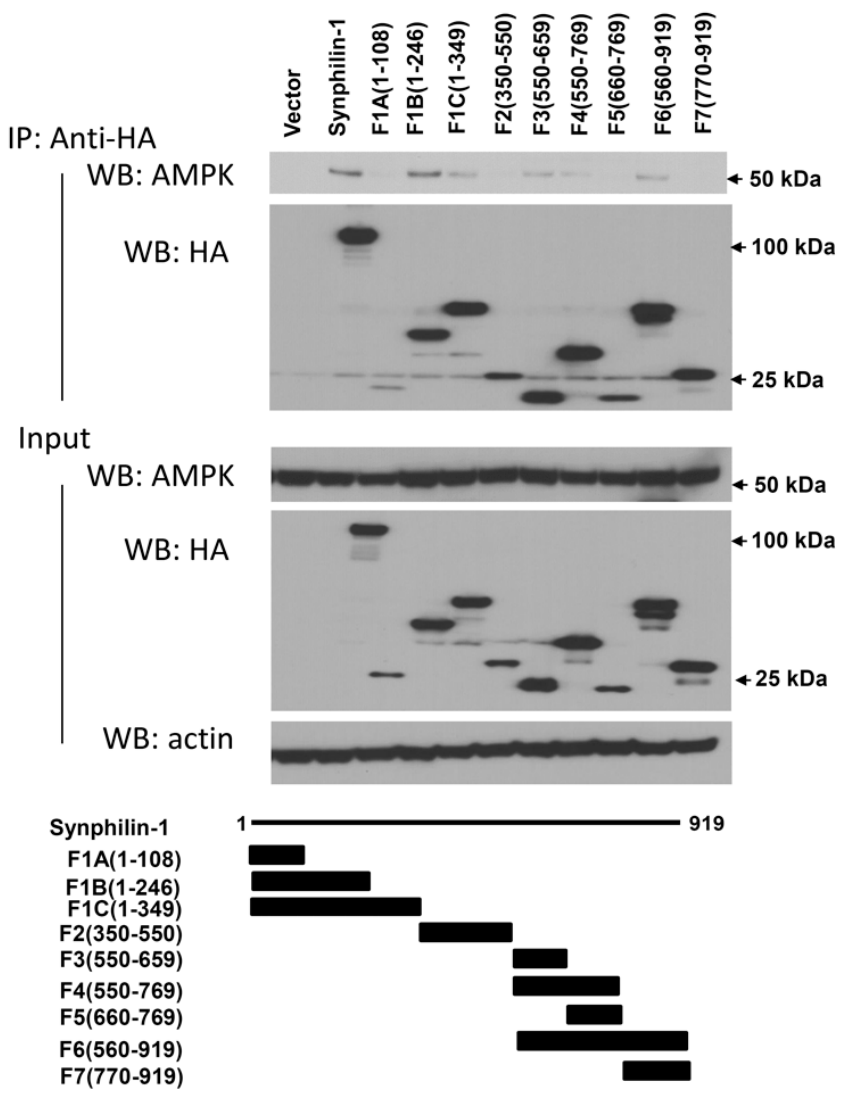

Figure 3. Map of the region of synphilin-1 interacting with AMPK. Lysates prepared from HEK 293 cells transfected with HA-tagged human full length or truncated synphilin-1 constructs were subjected to IP with anti-HA antibody, followed by anti-AMPK and anti-HA immunoblotting. The input of equal protein loading from cell lysates was shown by Western blot using anti-AMPK, anti-synphilin-1, and anti-actin antibodies.

\subsection{Knockdown of Synphilin-1 Reduced AMPK Phosphorylation}

On one hand, reduction of synphilin-1 expression by siRNA significantly attenuated AMPK phosphorylation, compared with cells expressing random control RNA (Figure 4). Whereas on the other hand, treatment with compound C, an AMPK inhibitor, significantly reduced synphilin-1 binding with AMPK (Figure 5A,B). Moreover, compound C reduced synphilin-1-induced AMPK phosphorylation (Figure 5A,C). Our results demonstrated that synphilin-1 mediated AMPK activation, while AMPK activity also regulated the interactions between synphilin-1 and AMPK. These findings suggest that synphilin-1 coupled with AMPK and had interacting effects on each other to regulate cellular activities. 


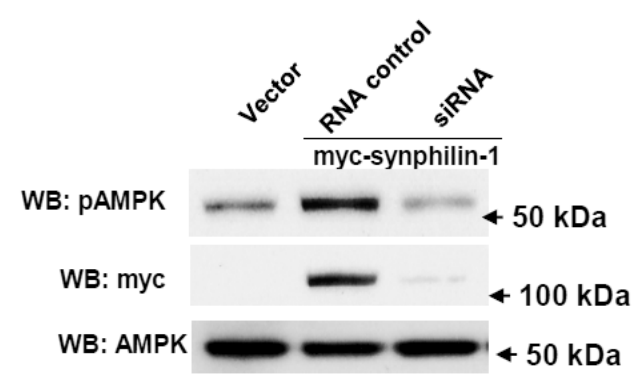

Figure 4. Knockdown of synphilin-1 reduced AMPK phosphorylation. Cells expressing synphilin-1 or vector were transiently transfected with siRNA targeting human synphilin-1 for three days. The cell lysates were subjected to Western blot using anti-myc, anti-phospho-AMPK $\alpha$, and anti-AMPK $\alpha$ antibodies. Representative blots from three separated experiments.

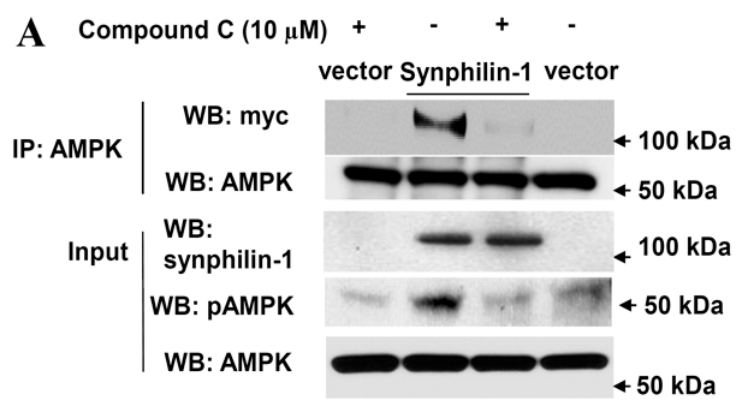

B

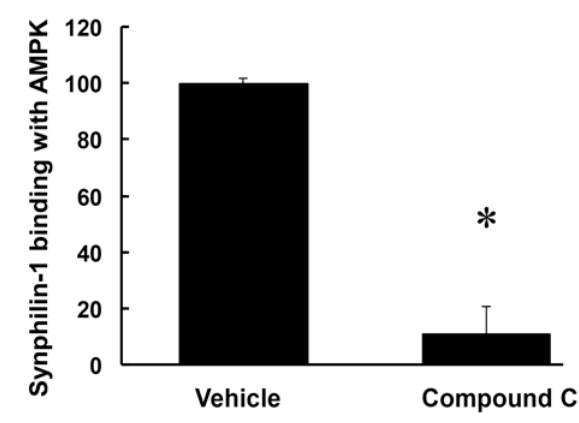

C

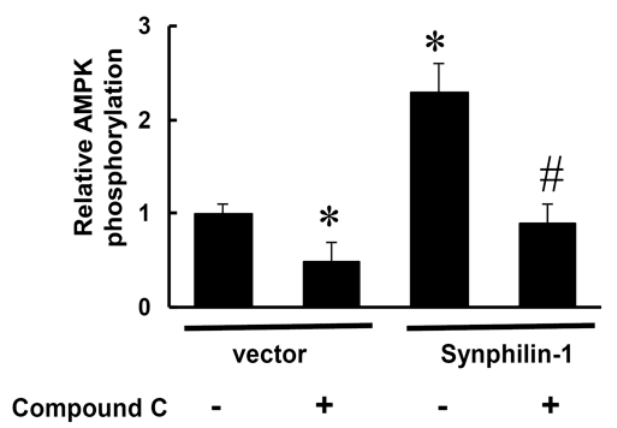

Figure 5. Compound C reduced synphilin-1 binding with AMPK. HEK293 cells were transfected with myc-synphilin-1 and vector, and treated with compound C $(10 \mu \mathrm{M})$ or vehicle for $48 \mathrm{~h}$. Cell lysates were subjected to co-IP using anti-AMPK $\alpha$ antibodies. IP samples and input cell lysates were subjected to Western blot analysis using anti-myc, anti-synphilin-1, anti-phospho-AMPK $\alpha$, and anti-AMPK $\alpha$ antibodies. (A). Representative blots. (B). Quantification of synphilin-1 binding with AMPK * $p<0.05$ by ANOVA followed by Tukey's post-hoc test, vs. cells expressing synphilin- 1 with vehicle treatment. (C). Quantification of AMPK phosphorylation levels normalized to total AMPK levels. $p<0.05$ by ANOVA followed by Tukey's post-hoc test, ${ }^{*}$ vs. vector cells with vehicle treatment; \# vs. cells expressing synphilin-1 with vehicle treatment. 


\subsection{Compound C Attenuated Synphilin-1-Induced Cellular ATP Increase}

ATP is the major cellular molecule responsible for providing energy for various cellular processes [4]. AMPK regulates systemic and cellular energy status, and functions as an energy sensor to monitor cellular energy status (ATP levels) in response to nutritional changes [20-24]. Consistent with our previous findings, synphilin-1 increased cellular ATP levels (Figure 6), while treatment with compound C, an AMPK inhibitor $(10 \mu \mathrm{M})$, attenuated synphilin-1-induced cellular ATP increase (Figure 6). These results indicate that inhibition of AMPK signaling modulates synphilin-1-induced increase in cellular ATP levels.

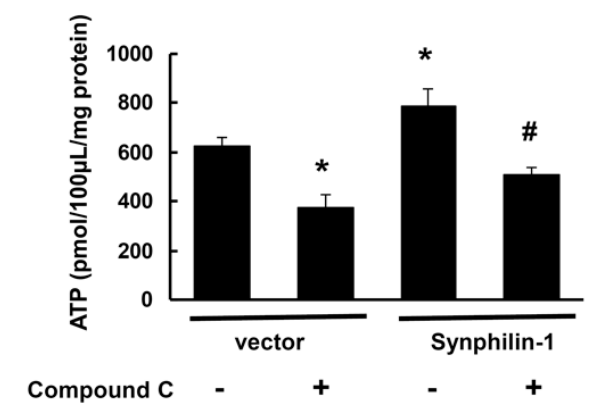

Figure 6. Compound $C$ reduced synphilin-1-induced cellular ATP increase. HEK293 cells were transfected with myc-synphilin-1 and vector and treated with compound C $(10 \mu \mathrm{M})$ or vehicle for $48 \mathrm{~h}$. Cells lysates were subjected to ATP assays. ${ }^{*} p<0.05$ by ANOVA followed by Tukey's post-hoc test, vs. vector control cells with vehicle treatment; $\# p<0.05$ by ANOVA, vs. cells expressing synphilin- 1 with vehicle treatment.

\section{Discussion}

In this study, we found that synphilin-1 interacted with AMPK, increased AMPK phosphorylation, and resulted in altered activity of the downstream signaling of ACC and p70S6. Knockdown of synphilin-1 reduced AMPK phosphorylation, while inhibition of AMPK by compound C reduced synphilin-1 and AMPK interactions. Moreover, compound $C$ also reduced the amount of synphilin-1-induced AMPK phosphorylation, and cellular ATP increase. These findings indicate that the interaction of synphilin-1 and AMPK play critical roles in regulating cellular energy balance. To our knowledge, this is the first report showing that synphilin-1 alters AMPK signaling, thereby regulating cellular energy status.

Synphilin-1 is a cytoplasmic protein that was cloned over a decade ago [3]. Synphilin-1 was originally identified in interactions with alpha-synuclein and has implications in the pathogenesis of Parkinson's disease related to protein aggregation and neuroprotection $[3,4,7-9,19,33]$. Recently, we and others have found that overexpression of synphilin- 1 increases food intake and body weight in mice [1]. Moreover, synphilin-1 overexpression also results in increases in food intake and body fat storage in flies [2]. In cultured cells, expression of synphilin-1 increases cellular ATP levels [34]. However, the underlying mechanisms of synphilin-1-induced positive energy balance have been unclear. In this study, our results showed that synphilin-1 bound with AMPK predominantly through two regions: $108-246$ aa and 550-769 aa. The region 108-246 aa is the ANK-repeat region, which contains a predicted protein-protein interaction domain that has been shown previously to bind with other proteins $[3,5,19]$. These findings strongly suggest a functional interaction between synphilin-1 and AMPK.

AMPK regulates systemic and cellular energy status, and functions as an energy sensor to monitor cellular energy status in response to nutritional changes [20-24]. AMPK regulates both energy-consuming anabolic pathways and energy-producing catabolic pathways [24,25,35]. AMPK can promote ATP production by increasing nutrient catabolism through regulation of key enzymes at the protein regulation level, while conserving ATP by regulating gene transcription to switch off 
biosynthetic pathways. ATP is the major energy currency of the cell, and is generated from catabolic pathways (such as fatty acid oxidation and glycolysis) [20-23,25,35]. ATP provides the energy needed for a variety of cellular activities.

Our results showed that overexpression of synphilin-1 increased AMPK phosphorylation (activation) compared with levels in vector control cells, while knockdown of synphilin-1 attenuated this increase. Moreover, synphilin-1 action on AMPK altered two major downstream targets of the AMPK pathway (ACC and p70S6K) and increased cellular ATP levels. We found that synphilin-1 decreased ACC phosphorylation, in accord with prior reports, which is involved in ATP production by promoting fatty acid oxidation and preventing fatty acid synthesis [25,32]. We also found that synphilin-1 increased the phosphorylation of serine/threonine kinase p70S6K, in accord with prior reports [36-38], which is related to maintaining ATP levels by regulating glucose homeostasis and protein synthesis [28], and promoting glucose intake, transport, and glycolytic activity [26]. Our results showed that inhibition of AMPK activity by compound C significantly reduced synphilin- 1 binding with AMPK, and attenuated synphilin-1-induced increases in cellular ATP levels. How synphilin-1 activates AMPK remains to be further investigated. One possibility is that synphilin-1 could compete with prolyl-isomerase Pin1 binding AMPK, thereby reducing the interactions between AMPK and Pin1. Previous studies report that Pin1 can bind synphilin-1 and AMPK [33,39]. Pin1 interacts with AMPK and downregulates AMPK activation [39]. Thus, decreased interaction between AMPK and Pin1 and increased interaction between synphilin-1 and Pin1 could result in an increase in AMPK activation. Taken together, these findings indicate that activation of AMPK by synphilin-1 regulates cellular ATP levels.

Synphilin-1-induced AMPK-mediated increases in cellular ATP levels could contribute to two types of cellular activities. One is to provide ample energy for various cellular functions, such as cell growth and protection against toxic stimuli [19]. Previous studies have shown that synphilin-1 has a neurotrophic effect and protects against mutant- $\alpha$-synuclein and rotenone-induced toxicity in PD models [3,4,7-9]. Moreover, increases in cellular ATP levels may also help the sequestration or clearance of toxic proteins by promoting the interactions between synphilin- 1 and other proteins (e.g., $\alpha$-synuclein and parkin) in forming protein aggregates [3,4,7-9]. The other potential type of cellular activity of synphilin-1-induced AMPK activation is to augment the energy storage pathways by promoting lipid and glycogen synthesis, which may contribute to fat and glycogen accumulation and deposition [19]. In line with this notion, we previously demonstrated that expression of synphilin-1 induces insulin resistance and increases triglyceride levels and fat storage both in flies and in mice $[1,2]$. Thus, the increases of cellular ATP levels more likely contribute to the obese-like phenotypes in synphilin-1 transgenic mice and flies [1,2].

In summary, these studies reveal novel cellular functions of synphilin-1 in regulating AMPK-linked signaling pathways, thereby contributing to increases in cellular ATP levels. These findings provide a new molecular basis for understanding synphilin-1 biology and the roles of synphilin-1 in regulating energy homeostasis.

\section{Materials and Methods}

\subsection{Reagents}

Media for cell culture, anti-hemagglutinin (HA) polyclonal antibody, and LipofectAMINE Plus reagent were ordered from Invitrogen (Carlsbad, CA, USA). Anti-AMPK $\alpha$ (AMPK $\alpha$-subunit), anti-phosphorylated AMPK $\alpha$ (Thr 172), anti-ACC $\alpha$, anti-phospho-ACC $\alpha$, anti-p70S6, and anti-phospho-p70S6 antibodies were purchased from Cell Signaling Technology (Beverly, MA, USA). Monoclonal anti-HA was from Roche Molecular Biochemicals (Indianapolis, IN, USA). The anti-myc antibody and the polyclonal anti-HA antibodies were from Santa Cruz Biotechnology (Santa Cruz, CA, USA). The anti-human synphilin-1 polyclonal antibody was generated using the human synphilin-1 
fragment (34-500 aa) as the antigen as previously described [3]. Anti-actin antibody was from Sigma (St. Louis, MO, USA). Compound C (an AMPK inhibitor) [40] was from Sigma (Burlington, MA, USA).

\subsection{Cell Culture, Plasmids, and siRNA Transfection}

The myc- or HA-tagged human full-length cDNA are in the pRK5 vector under CMV promoter as described previously $[7,13]$. Human synphilin-1 fragment constructs were provided by Drs. Christopher A. Ross and S. Engelender [7,13]. Human HEK293 cells were ordered from ATCC and grown in Dulbecco's modified Eagle's medium (DMEM; high glucose; Invitrogen) with 10\% fetal bovine serum (FBS) and 1\% antibiotics (Invitrogen). Transient transfections of various constructs were performed using LipofectAMINE Plus (Invitrogen) according to the manufacturer's protocol. The specific siRNA targeting synphilin-1 and its scrambled RNA control sequence were purchased from Dharmacon (Chicago, IL, USA). siRNAs were transfected into cells for $72 \mathrm{~h}$ using LipofectAMINE 2000 according to the manufacturer's protocol. Cells were harvested for immunoprecipitation (IP) and Western blot analysis.

\subsection{Immunoprecipitation (IP), GST Pull-Down and Western Blot Analysis}

Cells were harvested in lysis buffer (Cell Signaling) with protease inhibitors $(10 \mu \mathrm{g} / \mathrm{mL}$ aprotinin/ $5 \mathrm{mM}$ PMSF $/ 10 \mu \mathrm{g} / \mathrm{mL}$ pepstatin $/ 10 \mu \mathrm{g} / \mathrm{mL}$ leupeptin) as described previously [16]. The resulting lysates were subjected to IP and Western blot analysis. IP experiments were performed using anti-myc, anti-HA, or anti-AMPK antibodies and protein G Plus/protein A-agarose (GE, Pittsburgh, PA USA) as described previously [7]. GST pull-down was performed as described previously [3]. For Western blot analysis, proteins from each sample were separated on 4-12\% NuPAGE Bis-Tris gels, and transferred onto polyvinylidene difluoride membranes (PVDF, Invitrogen) for $3 \mathrm{~h}$. Then the gels were stained with Coomassie blue to assure transfer efficiency. The PVDF membranes were blocked with $5 \%$ non-fat milk and incubated with various primary antibodies as listed in Section 4.1 for 1 to $4 \mathrm{~h}$ at room temperature. The goat anti-mouse or goat anti-rabbit horseradish-peroxidase conjugated antibodies were used as secondary detection antibodies for $1 \mathrm{~h}$ of incubation. Enhanced chemiluminescence reagents (PerkinElmer, Waltham, MA, USA) were incubated for $1 \mathrm{~min}$ to detect the proteins in PVDF membranes.

\subsection{ATP Assay}

Cells were harvested in a lysis buffer (Cell Signaling Technology). Lysates were subjected to protein assays using a Bradford protein assay kit (Sigma St. Louis, MO, USA) according to the manufacturer's protocol. An aliquot of $10 \mu \mathrm{g}$ of protein from each sample was subjected to ATP luminescent assays using an ATP Determination Kit (Invitrogen) according to the manufacturer's protocol. The experiments were repeated three times in duplicate.

\subsection{Data Analysis}

Quantitative data were represented as means \pm Standard Error of the Mean (SEM). Protein and ATP levels were used for one-way or two-way analysis of variance (ANOVA) by Sigmastat 3.1 statistical software (Aspire Software International, Leesburg, VA, USA). Post-ANOVA analysis of group differences was performed with the Tukey test. A $p$ value $<0.05$ was considered significant.

Author Contributions: Conception and design of the study: T.L., W.W.S., T.H.M.; acquisition, analysis, and interpretation of data: T.L., J.L., G.G., B.N., X.L., G.Z., D.Y., T.H.M., W.W.S. Drafting the manuscript: T.L., W.W.S., T.H.M.; statistical analysis: T.L., W.W.S. All authors have read and agreed to the published version of the manuscript.

Funding: This work was supported by National Institutes of Health, Grants: DK083410 to W.W.S.

Acknowledgments: We thank C.A. Ross and S. Engelender for providing the human synphilin-1 fragment constructs. 
Conflicts of Interest: All authors declare no conflicts of interest.

Data sharing statement: The data that support the findings of this study are all included in this manuscript.

\section{References}

1. Li, X.; Tamashiro, K.L.K.; Liu, Z.; Bello, N.T.; Wang, X.; Aja, S.; Bi, S.; Ladenheim, E.E.; Ross, C.A.; Moran, T.H.; et al. A novel obesity model: Synphilin-1-induced hyperphagia and obesity in mice. Int. J. Obes. 2012, 36, 1215-1221. [CrossRef] [PubMed]

2. Liu, J.; Li, T.; Yang, D.; Ma, R.; Moran, T.H.; Smith, W.W. Synphilin-1 alters metabolic homeostasis in a novel Drosophila obesity model. Int. J. Obes. 2012, 36, 1529-1536. [CrossRef] [PubMed]

3. Engelender, S.; Kaminsky, Z.; Xin, G.; Sharp, A.H.; Amaravi, R.K.; Kleiderlein, J.J.; Margolis, R.L.; Troncoso, J.C.; Lanahan, A.A.; Worley, P.F.; et al. Synphilin-1 associates with $\alpha$-synuclein and promotes the formation of cytosolic inclusions. Nat. Genet. 1999, 22, 110-114. [CrossRef]

4. Ribeiro, C.S.; Carneiro, K.; Ross, C.A.; Menezes, J.R.L.; Engelender, S. Synphilin-1 is developmentally localized to synaptic terminals, and its association with synaptic vesicles is modulated by $\alpha$-synuclein. $J$. Biol. Chem. 2002, 277, 23927-23933. [CrossRef] [PubMed]

5. Nagano, Y.; Yamashita, H.; Takahashi, T.; Kishida, S.; Nakamura, T.; Iseki, E.; Hattori, N.; Mizuno, Y.; Kikuchi, A.; Matsumoto, M. Siah-1 Facilitates Ubiquitination and Degradation of Synphilin-1. J. Biol. Chem. 2003, 278, 51504-51514. [CrossRef] [PubMed]

6. Alvarez-Castelao, B.; Castaño, J.G. Synphilin-1 inhibits alpha-synuclein degradation by the proteasome. Cell. Mol. Life Sci. 2011, 68, 2643-2654. [CrossRef]

7. Smith, W.W.; Margolis, R.L.; Li, X.; Troncoso, J.C.; Lee, M.K.; Dawson, V.L.; Dawson, T.M.; Iwatsubo, T.; Ross, C.A. $\alpha$-synuclein phosphorylation enhances eosinophilic cytoplasmic inclusion formation in SH-SY5Y cells. J. Neurosci. 2005, 25, 5544-5552. [CrossRef]

8. Chung, K.K.K.; Zhang, Y.; Lim, K.L.; Tanaka, Y.; Huang, H.; Gao, J.; Ross, C.A.; Dawson, V.L.; Dawson, T.M. Parkin ubiquitinates the $\alpha$-synuclein-interacting protein, synphilin-1: Implications for Lewy-body formation in Parkinson disease. Nat. Med. 2001, 7, 1144-1150. [CrossRef]

9. Wakabayashi, K.; Engelender, S.; Yoshimoto, M.; Tsuji, S.; Ross, C.A.; Takahashi, H. Synphilin-1 is present in Lewy bodies in Parkinson's disease. Ann. Neurol. 2000, 47, 521-523. [CrossRef]

10. Szargel, R.; Rott, R.; Engelender, S. Synphilin-1 isoforms in Parkinson's disease: Regulation by phosphorylation and ubiquitylation. Cell. Mol. Life Sci. 2008, 65, 80-88. [CrossRef]

11. O’Farrell, C.; Murphy, D.D.; Petrucelli, L.; Singleton, A.B.; Hussey, J.; Farrer, M.; Hardy, J.; Dickson, D.W.; Cookson, M.R. Transfected synphilin-1 forms cytoplasmic inclusions in HEK293 cells. Mol. Brain Res. 2001, 97, 94-102. [CrossRef]

12. Lee, G.; Tanaka, M.; Park, K.; Lee, S.S.; Kim, Y.M.; Junn, E.; Lee, S.H.; Mouradian, M.M. Casein Kinase II-mediated Phosphorylation Regulates $\alpha$-Synuclein/Synphilin-1 Interaction and Inclusion Body Formation. J. Biol. Chem. 2004, 279, 6834-6839. [CrossRef]

13. Avraham, E.; Szargel, R.; Eyal, A.; Rott, R.; Engelender, S. Glycogen synthase kinase $3 \beta$ modulates synphilin-1 ubiquitylation and cellular inclusion formation by SIAH: Implications for proteasomal function and lewy body formation. J. Biol. Chem. 2005, 280, 42877-42886. [CrossRef]

14. Marx, F.P.; Soehn, A.S.; Berg, D.; Melle, C.; Schiesling, C.; Lang, M.; Kautzmann, S.; Strauss, K.M.; Franck, T.; Engelender, S.; et al. The proteasomal subunit S6 ATPase is a novel synphilin-1 interacting protein-implications for Parkinson's disease. Faseb J. 2007, 21, 1759-1767. [CrossRef]

15. Smith, W.W.; Liu, Z.; Liang, Y.; Masuda, N.; Swing, D.A.; Jenkins, N.A.; Copeland, N.G.; Troncoso, J.C.; Pletnikov, M.; Dawson, T.M.; et al. Synphilin-1 attenuates neuronal degeneration in the A53T $\alpha$-synuclein transgenic mouse model. Hum. Mol. Genet. 2010, 19, 2087-2098. [CrossRef]

16. Li, X.; Liu, Z.; Tamashiro, K.; Shi, B.; Rudnicki, D.D.; Ross, C.A.; Moran, T.H.; Smith, W.W. Synphilin-1 exhibits trophic and protective effects against Rotenone toxicity. Neuroscience 2010, 165, 455-462. [CrossRef]

17. Giaime, E.; Sunyach, C.; Herrant, M.; Grosso, S.; Auberge, P.; McLean, P.J.; Checler, F.; Alves Da Costa, C. Caspase-3-derived C-terminal product of synphilin-1 displays antiapoptotic function via modulation of the p53-dependent cell death pathway. J. Biol. Chem. 2006, 281, 11515-11522. [CrossRef] 
18. Hernández-Vargas, R.; Fonseca-Ornelas, L.; López-González, I.; Riesgo-Escovar, J.; Zurita, M.; Reynaud, E. Synphilin suppresses $\alpha$-synuclein neurotoxicity in a Parkinson's disease Drosophila model. Genesis 2011, 49, 392-402. [CrossRef]

19. Liu, J.; Li, T.; Thomas, J.M.; Pei, Z.; Jiang, H.; Engelender, S.; Ross, C.A.; Smith, W.W. Synphilin-1 attenuates mutant LRRK2-induced neurodegeneration in Parkinson's disease models. Hum. Mol. Genet. 2016, 25, 672-680. [CrossRef]

20. Kola, B.; Grossman, A.B.; Korbonits, M. The role of AMP-activated protein kinase in obesity. Front. Horm. Res. 2008, 36, 198-211.

21. Misra, P. AMP activated protein kinase: A next generation target for total metabolic control. Expert Opin. Targets 2008, 12, 91-100. [CrossRef]

22. Hue, L.; Rider, M.H. The AMP-activated protein kinase: More than an energy sensor. Essays Biochem. 2007, 43, 121-137. [PubMed]

23. Winder, W.W.; Thomson, D.M. Cellular energy sensing and signaling by AMP-activated protein kinase. Cell Biochem. Biophys. 2007, 47, 332-347. [CrossRef] [PubMed]

24. Ruderman, N.B.; Carling, D.; Prentki, M.; Cacicedo, J.M. AMPK, insulin resistance, and the metabolic syndrome. J. Clin. Investig. 2013, 123, 2764-2772. [CrossRef] [PubMed]

25. Ke, R.; Xu, Q.; Li, C.; Luo, L.; Huang, D. Mechanisms of AMPK in the maintenance of ATP balance during energy metabolism. Cell Biol. Int. 2018, 42, 384-392. [CrossRef]

26. Carling, D. The role of the AMP-activated protein kinase in the regulation of energy homeostasis. Novartis Found. Symp. 2007, 286, 72-81.

27. Kahn, B.B.; Alquier, T.; Carling, D.; Hardie, D.G. AMP-activated protein kinase: Ancient energy gauge provides clues to modern understanding of metabolism. Cell Metab. 2005, 1, 15-25. [CrossRef]

28. Xue, B.; Kahn, B.B. AMPK integrates nutrient and hormonal signals to regulate food intake and energy balance through effects in the hypothalamus and peripheral tissues. J. Physiol. 2006, 574, 73-83. [CrossRef]

29. Minokoshi, Y.; Alquier, T.; Furukawa, N.; Kim, Y.-B.; Lee, A.; Xue, B.; Mu, J.; Foufelle, F.; Ferre, P.; Birnbaum, M.J.; et al. AMP-kinase regulates food intake by responding to hormonal and nutrient signals in the hypothalamus. Nature 2004, 428, 569-574. [CrossRef]

30. Andersson, U.; Filipsson, K.; Abbott, C.R.; Woods, A.; Smith, K.; Bloom, S.R.; Carling, D.; Small, C.J. AMP-activated Protein Kinase Plays a Role in the Control of Food Intake. J. Biol. Chem. 2004, 279, 12005-12008. [CrossRef]

31. Moran, T.H.; Gao, S. Looking for food in all the right places? Cell Metab. 2006, 3, 233-234. [CrossRef]

32. Gao, S.; Kinzig, K.P.; Aja, S.; Scott, K.A.; Keung, W.; Kelly, S.; Strynadka, K.; Chohnan, S.; Smith, W.W.; Tamashiro, K.L.K.; et al. Leptin activates hypothalamic acetyl-CoA carboxylase to inhibit food intake. Proc. Natl. Acad. Sci. USA 2007, 104, 17358-17363. [CrossRef]

33. Ryo, A.; Togo, T.; Nakai, T.; Hirai, A.; Nishi, M.; Yamaguchi, A.; Suzuki, K.; Hirayasu, Y.; Kobayashi, H.; Perrem, K.; et al. Prolyl-isomerase Pin1 accumulates in Lewy bodies of Parkinson Disease and facilitates formation of $\alpha$-synuclein inclusions. J. Biol. Chem. 2006, 281, 4117-4125. [CrossRef]

34. Li, T.; Liu, J.; Smith, W.W. Synphilin-1 binds ATP and regulates intracellular energy status. PLoS ONE 2014, 9, e115233. [CrossRef]

35. Hardie, D.G. AMP-activated/SNF1 protein kinases: Conserved guardians of cellular energy. Nat. Rev. Mol. Cell Biol. 2007, 8, 774-785. [CrossRef]

36. Inoki, K.; Zhu, T.; Guan, K.-L. TSC2 mediates cellular energy response to control cell growth and survival. Cell 2003, 115, 577-590. [CrossRef]

37. Hardie, D.G. AMPK and Raptor: Matching Cell Growth to Energy Supply. Mol. Cell 2008, 30, $263-265$. [CrossRef]

38. Yang, X.; Yang, C.; Farberman, A.; Rideout, T.C.; de Lange, C.F.; France, J.; Fan, M.Z. The mammalian target of rapamycin-signaling pathway in regulating metabolism and growth. J. Anim. Sci. 2008, 86, 36-50. [CrossRef] 
39. Khanal, P.; Kim, G.; Yun, H.J.; Cho, H.G.; Choi, H.S. The prolyl isomerase Pin1 interacts with and downregulates the activity of AMPK leading to induction of tumorigenicity of hepatocarcinoma cells. Mol. Carcinog. 2013, 52, 813-823. [CrossRef]

40. Rao, E.; Zhang, Y.; Li, Q.; Hao, J.; Egilmez, N.K.; Suttles, J.; Li, B. AMPK-dependent and independent effects of AICAR and compound C on T-cell responses. Oncotarget 2016, 7, 33783-33795. [CrossRef]

(C) 2020 by the authors. Licensee MDPI, Basel, Switzerland. This article is an open access article distributed under the terms and conditions of the Creative Commons Attribution (CC BY) license (http://creativecommons.org/licenses/by/4.0/). 